\title{
Conocimiento Didáctico-Matemático para la Enseñanza de la Matemática Elemental en futuros profesores de educación básica: diseño, construcción y validación de un instrumento de evaluación

\author{
Didactic-Mathematical Knowledge for the Teaching of Elementary Mathematics \\ in future teachers of basic education: design, construction and validation of an \\ evaluation instrument
}

\author{
Nataly Pincheira Hauck ${ }^{a}$, Claudia Vásquez Ortiz \\ ${ }^{a}$ Pontificia Universidad Católica de Chile, Campus Villarrica. \\ Correo electrónico: npincheirah@uc.cl \\ ${ }^{\text {b }}$ Pontificia Universidad Católica de Chile, Campus Villarrica. \\ Correo electrónico: cavasque@uc.cl.
}

\begin{abstract}
RESUMEN
En este trabajo se presenta el proceso de diseño, construcción y validación de un instrumento cuyo propósito es evaluar aspectos parciales del conocimiento didáctico-matemático para la enseñanza de las matemáticas elementales en futuros profesores de Educación Básica. Con esta finalidad, nos hemos situado desde la perspectiva del Enfoque Ontosemiótico del Conocimiento y la instrucción Matemática, pues éste brinda herramientas de análisis que permiten aportar evidencias sobre el conocimiento común del contenido, el conocimiento ampliado del contenido y el conocimiento especializado del contenido para la mejora de la formación inicial docente.

Palabras claves: formación de profesores, enfoque ontosemiótico, enseñanza de la matemática.
\end{abstract}

\begin{abstract}
This paper presents the process of design, construction and validation of an instrument whose purpose is to evaluate partial aspects of didactic-mathematical knowledge for the teaching of elementary mathematics in future teachers of Basic Education. To this end, we have placed ourselves from the perspective of the Onto-semiotic Approach to Knowledge and Mathematical Instruction, since it provides analysis tools that allow us to provide evidence about the common knowledge of the content, the expanded knowledge of the content and the specialized knowledge of the content for the improvement of initial teacher training.
\end{abstract}

Keywords: teacher training, onto-semiotic approach, teaching mathematics. 


\section{INTRODUCCIÓN}

La destacada importancia de la formación inicial docente en la calidad de la educación, ha sido parte de la preocupación por mejorar la preparación de los futuros profesores, especialmente en el área de la matemática, particularmente si consideramos los bajos resultados obtenidos por nuestro país tanto en evaluaciones internacionales como nacionales que refieren sobre la calidad de los egresados de las carreras de pedagogía en Educación Básica. Un ejemplo de esto es el estudio comparativo internacional Teacher Education and Development Study in Mathematics TEDS-M (Tatto, Schwille, Ingvarson, Rowley y Peck 2012), que evalúa la formación inicial docente de los maestros de primaria y secundaria en matemáticas, ubicando a Chile en penúltimo lugar. De igual manera, la prueba INICIA (2014) evidencia grandes vacíos en el ámbito disciplinar y pedagógico que poseen los egresados de las carreras de pedagogía en Educación Básica. Es en este contexto, que surge la necesidad de contar con instrumentos que permitan analizar el conocimiento didáctico-matemático que poseen los futuros profesores de Educación Básica para enseñar matemáticas elementales.

Para este estudio, entenderemos por matemática elemental desde la perspectiva de Ma, quien reconoce que los conocimientos del profesor son fundamentales en la tarea de enseñar matemática, destacando el concepto de comprensión profunda de las matemáticas elementales para identificar los conocimientos que debería poseer un profesor para ejercer en plenitud su tarea de enseñar matemáticas elementales a niños y niñas, en los primeros años de escolaridad (Ma 1999). Es decir, desde la mirada de Liping Ma, ¿poseen los futuros profesores de Educación General Básica una comprensión profunda de la matemática que deberán enseñar y sobre cómo enseñarla?

Este estudio da a conocer el proceso de diseño, construcción y validación de un cuestionario que permite evaluar aspectos iniciales relevantes del conocimiento didácticomatemático que poseen los futuros profesores de Educación Básica para la enseñanza de las matemáticas elementales y desde esta perspectiva poder diagnosticar y comprender las necesidades formativas tanto didácticas como disciplinares, con el propósito de contar con información útil sobre la calidad de la formación inicial docente, que permita centrarse en el avance y fortalecimiento de las bases de su estructura para mejorar la futura práctica docente.

Para la elaboración del instrumento se consideró el Modelo de Conocimiento DidácticoMatemático (CDM) que se fundamenta en el Enfoque Ontosemiótico del Conocimiento y la Instrucción Matemática (EOS) (Godino 2002; Godino, Batanero y Font 2007).

\section{FUNDAMENTACIÓN TEÓRICA}

\subsection{CONOCIMIENTO DIDÁCTICO-MATEMÁTICO PARA LA ENSEÑANZA DE LA MATEMÁTICA ELEMENTAL}

En la actualidad son variados los modelos de conocimiento matemático que aportan en la formación de profesores, sin embargo, en algunos casos estos resultan ser de carácter general y no atienden a un análisis detallado de los tipos de conocimientos que debiese tener un profesor para lograr una enseñanza eficaz de las matemáticas. Bajo esta mirada es 
que Godino, a través de una reestructuración de algunas perspectivas teóricas tales como, las nociones teóricas del Enfoque Ontosemiótico (Godino 2002; Godino et al. 2007), la noción de proficiencia en la enseñanza de las matemáticas (Schoenfeld y Kilpatrick 2008) y el modelo del conocimiento matemático para la enseñanza (MKT) (Hill, Ball y Schilling 2008), propone un modelo integrador para el análisis y desarrollo del conocimiento didáctico-matemático, basado en un sistema de categorías. Dicho modelo, considera herramientas concretas que permiten un análisis minucioso del conocimiento didácticomatemático del profesor, incluyendo seis facetas o dimensiones (epistemológica, cognitiva, afectiva, interaccional, mediacional y ecológica) implicadas en el proceso de enseñanza y aprendizaje que un profesor deba poner en práctica para la enseñanza de temas específicos de matemáticas (Figura 1).

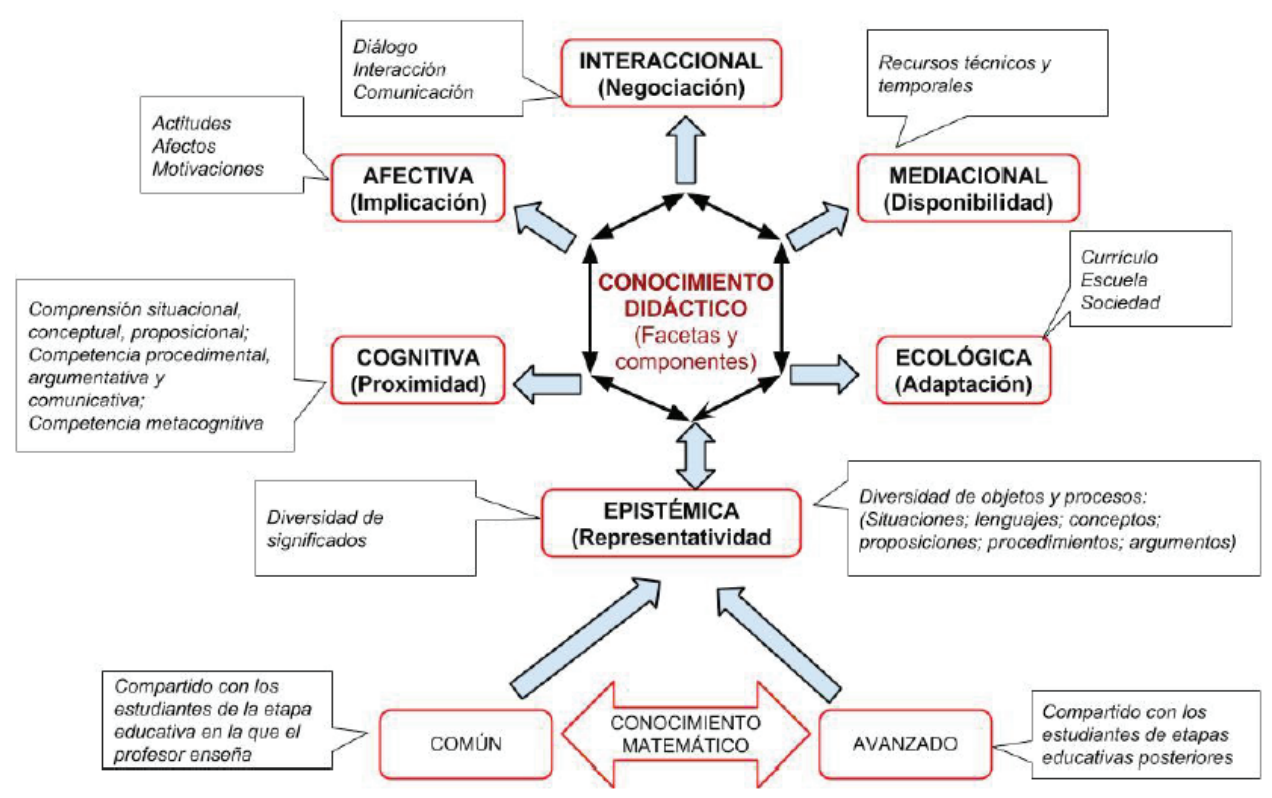

Figura 1. Conocimiento Didáctico-Matemático basado en el EOS

Este modelo se fundamenta en las siguientes seis facetas (Godino 2009):

- Epistémica: Conocimientos matemáticos relativos al contexto institucional en que se realiza el proceso de estudio y la distribución en el tiempo de los diversos componentes del contenido (problemas, lenguajes, procedimientos, definiciones, propiedades, argumentos).

- Cognitiva: Conocimientos personales de los estudiantes y progresión de los aprendizajes.

- Afectiva: Estados afectivos (actitudes, emociones, creencias, valores) de cada alumno con relación a los objetivos matemáticos y al proceso de estudio seguido. 
- Mediacional: Recursos tecnológicos y asignación del tiempo a las distintas acciones y procesos.

- Interaccional: Patrones de interacción entre el profesor y los estudiantes y su secuencia orientada a la fijación y negociación de los significados.

- Ecológica: Sistemas de relaciones con el entorno social, político, económico, etc., que soporta y condiciona el proceso de estudio.

Así, el conocimiento matemático se compone del conocimiento común, que corresponde al conocimiento que ha recibido un estudiante durante su etapa educativa, mientras que el conocimiento avanzado, se adquiere en etapas educativas posteriores. Ambos tipos de conocimientos están incorporados en la faceta epistémica, desde donde se puede analizar el conocimiento matemático. Por otra parte, la faceta cognitiva y afectiva tienen relación con los estudiantes, las facetas interaccional y mediacional con la enseñanza, mientras que la ecológica se relaciona con el currículo.

Más tarde, Godino y Pino-Fan reinterpretan las premisas iniciales del EOS y presentan una reestructuración de los componentes del MKT (Godino y Pino-Fan 2013), generando un vínculo entre éstos y las seis facetas mencionadas anteriormente. En base a esta reestructuración es que Pino-Fan, Godino y Font proponen tres categorías globales sobre el conocimiento didáctico-matemático (Pino-Fan, Godino y Font 2013):

a) conocimiento común del contenido: este conocimiento es analizado a través de la faceta epistémica, y hace referencia al conocimiento que el profesor debe poner en práctica para resolver situaciones problemáticas en relación con un tema específico matemático, no ligado necesariamente a la enseñanza.

b) conocimiento ampliado del contenido: este conocimiento se analiza a partir de la faceta epistémica, teniendo relación con el conocimiento que posee el profesor al plantear una cierta situación problema a sus estudiantes, teniendo la capacidad de vincularlo con otros contenidos del currículo.

c) conocimiento especializado: al igual que en los casos anteriores, este conocimiento se analiza desde la faceta epistémica y hace mención a aquel conocimiento adicional que debe manejar un profesor, lo cual lo diferencia de otra persona que no es un profesor, pero que conoce de matemática.

El conocimiento especializado a su vez, se subdivide en cuatro categorías:

- Conocimiento del contenido especializado: se relaciona con la capacidad que tiene un profesor de identificar el conocimiento presente en una determinada situación problemática en relación a un contenido. Se fundamenta en las facetas implicadas en los procesos de instrucción matemática.

- Conocimiento del contenido en relación con los estudiantes: este conocimiento se basa en la faceta cognitiva y afectiva, según Godino, tiene relación con la capacidad que tiene el profesor para describir los tipos de configuraciones cognitivas que presentan los estudiantes al resolver una situación problema, describir los conflictos de aprendizaje que presentan los estudiantes frente a una problemática, describir estrategias que puedan implementar los estudiantes para dar solución a diversas situaciones problemáticas (Godino 2009).

- Conocimiento del contenido en relación con la enseñanza: se basa en la faceta interaccional y mediacional, según Godino, está relacionada con la reflexión que 
realiza el profesor acerca de la enseñanza y el aprendizaje, y la identificación de las consecuencias que pueden tener sobre el aprendizaje los modelos de la clase (Godino 2009).

- Conocimiento del contenido en relación con el currículo: se basa en la faceta ecológica y tiene relación con el contexto donde se desarrolla el proceso de enseñanza aprendizaje.

Para evaluar y analizar el conocimiento didáctico-matemático que poseen los futuros profesores de educación general básica para enseñar matemáticas elementales, se a decidido diseñar, construir y validar un instrumento que permita evaluar aspectos parciales relacionados a los componentes del modelo propuesto por Godino y colaboradores. Dicho instrumento, tiene por objetivo recoger datos sobre el conocimiento didáctico-matemático en la enseñanza de la matemática elemental por futuros profesores, de manera tal, que permita generar evidencia respecto del conocimiento común del contenido, ampliado del contenido y especializado.

\subsection{PRESENCIA DE LA MATEMÁTICA ELEMENTAL EN LA FORMACIÓN DEL PROFESORADO}

La formación inicial docente cobra un rol primordial en materias de políticas educativas, ya que es un factor de alta incidencia en el logro de aprendizaje de los estudiantes. Por tanto, es importante contar con profesores bien formados, tanto en el área disciplinar, como en el área pedagógica, para lograr una enseñanza efectiva.

Para orientar los conocimientos y habilidades que se espera que maneje todo profesor al finalizar su formación inicial, el Ministerio de Educación de Chile ha elaborado un conjunto de estándares para egresados de carreras de educación básica, éstos describen lo que tienen que saber y saber hacer los estudiantes egresados de las carreras de Pedagogía en Educación Básica.

Dichos estándares se han organizado en base a dos grandes categorías, considerando las cuatro disciplinas (Lenguaje y comunicación; Matemática; Historia, Geografía y Ciencias Sociales; y Ciencias Naturales): estándares pedagógicos y estándares disciplinarios. Ambas categorías se articulan y complementan entre sí, con la finalidad de brindar al futuro profesor los conocimientos y habilidades necesarios para el desempeño de la docencia:

Estándares pedagógicos: relacionados con áreas de competencia genérica de la función docente (Tabla 1). Corresponde a los elementos que debe manejar un profesor para el correcto desarrollo del proceso de enseñanza, vale decir, conocimiento del currículo, diseño y evaluación del proceso de enseñanza aprendizaje, gestión de clases, interacción con los estudiantes, ambiente y organización de la sala de clases, entre otros, independiente de la disciplina que enseñe. 
Estudios Pedagógicos XLIV, $\mathrm{N}^{\circ}$ 1: 25-48, 2018

CONOCIMIENTO DIDÁCTICO-MATEMÁTICO PARA LA ENSEÑANZA DE LA MATEMÁTICA ELEMENTAL EN FUTUROS PROFESORES DE EDUCACIÓN BÁSICA: DISEÑO, CONSTRUCCIÓN Y VALIDACIÓN DE UN ...

Tabla 1. Estándares pedagógicos para egresados de carreras de pedagogía en educación básica.

\begin{tabular}{|c|c|}
\hline \multicolumn{2}{|r|}{ Estándares Pedagógicos } \\
\hline Estándar 1 & Conoce a los estudiantes de Educación Básica y sabe cómo aprenden. \\
\hline Estándar 2 & Está preparado para promover el desarrollo personal y social de los estudiantes. \\
\hline Estándar 3 & $\begin{array}{l}\text { Conoce el currículo de enseñanza básica y usa sus diversos instrumentos curriculares } \\
\text { para analizar y formular propuestas pedagógicas y evaluativas. }\end{array}$ \\
\hline Estándar 4 & $\begin{array}{l}\text { Sabe cómo diseñar e implementar estrategias de enseñanza aprendizaje, adecuadas } \\
\text { para los objetivos de aprendizaje y de acuerdo al contexto. }\end{array}$ \\
\hline Estándar 5 & $\begin{array}{l}\text { Está preparado para gestionar la clase y crear un ambiente apropiado para el } \\
\text { aprendizaje según contextos. }\end{array}$ \\
\hline Estándar 6 & $\begin{array}{l}\text { Conoce y sabe aplicar métodos de evaluación para observar el progreso de los } \\
\text { estudiantes y sabe usar los resultados para retroalimentar el aprendizaje y la práctica } \\
\text { pedagógica. }\end{array}$ \\
\hline Estándar 7 & Conoce cómo genera y transforma la cultura escolar. \\
\hline Estándar 8 & Está preparado para atender la diversidad y promover la integración en el aula. \\
\hline Estándar 9 & $\begin{array}{l}\text { Se comunica oralmente y por escrito de forma efectiva en diversas situaciones } \\
\text { asociadas al quehacer docente. }\end{array}$ \\
\hline Estándar 10 & $\begin{array}{l}\text { Aprende en forma continua y reflexiona sobre su práctica y su inserción en el sistema } \\
\text { educacional. }\end{array}$ \\
\hline
\end{tabular}

Estándares disciplinarios: considera los conocimientos y habilidades que debe demostrar un profesor desde la disciplina que enseña, tales como: diseño de estrategias de enseñanza e implementación de experiencias de aprendizaje, conocimiento acerca de cómo aprenden los estudiantes, evaluación y reflexión en torno a los logros de aprendizaje de sus estudiantes.

En la asignatura de matemáticas, desde las orientaciones que entregan los estándares que rigen la formación del profesorado podemos analizar la presencia de la matemática elemental que deben enseñar los futuros profesores de educación básica, la cual se organiza según los cuatro ejes de contenidos del currículo escolar chileno: Números, Geometría, Álgebra y Datos y Azar.

El eje de números: este eje constituye el centro del currículo escolar de matemática en la educación básica. Encontraremos aquí los conceptos fundamentales que componen los cimientos sobre el cual se organiza el conocimiento matemático de los niños y niñas a lo largo de los diversos niveles educacionales. Es por ello que el dominio que debiese poseer un profesor en esta materia resulta ser trascendental. Este eje propone seis estándares, como se muestra en la tabla 2, el primero de ellos está orientado al sistema de numeración decimal, los estándares dos y tres hacen referencia a las operaciones de adición, sustracción, multiplicación y división de números naturales, mientras que los estándares cuarto y quinto se destaca al trabajo con fracciones, decimales, porcentajes, razones y proporciones, 
finalmente el estándar seis está vinculado al dominio disciplinar que debiese poseer un profesor en esta materia.

Tabla 2. Estándares de matemática, eje números, para egresados de carreras de pedagogía en educación básica.

\begin{tabular}{|l|l|}
\hline \multicolumn{2}{|c|}{ Números } \\
\hline Estándar 1 & Es capaz de conducir el aprendizaje del sistema de numeración decimal. \\
\hline Estándar 2 & Es capaz de conducir el aprendizaje de la adición y sustracción de números naturales. \\
\hline Estándar 3 & $\begin{array}{l}\text { Es capaz de conducir el aprendizaje de la multiplicación y división de los números } \\
\text { naturales. }\end{array}$ \\
\hline Estándar 4 & Está preparado para conducir el aprendizaje de fracciones y decimales. \\
\hline Estándar 5 & Está preparado para conducir el aprendizaje de porcentajes, razones y proporciones. \\
\hline Estándar 6 & Demuestra competencia disciplinaria en el eje de números. \\
\hline
\end{tabular}

El eje de geometría: este eje se convierte en una de las ramas más antiguas de la matemática y resulta ser un aporte al desarrollo del pensamiento matemático de niños y niñas. El eje de geometría está compuesto por cinco estándares, como se muestra en la tabla 3. El primero de ellos, el número siete, está orientado a la visualización de cuerpos y figuras, reconocimiento, clasificación y estimación de sus dimensiones. El segundo estándar, el número ocho, se vincula al trabajo de figuras planas presentes en el currículo escolar, tales como, triángulos, paralelogramos y polígonos. El estándar número nueve se relaciona con las mediciones, orientados al trabajo con peso, masa y volumen respecto de la forma de un objeto, mediante la utilización de unidades estandarizadas y no estandarizadas. El cuarto estándar, el número diez aborda temas relacionados al cálculo de perímetro, área y volumen. Finalmente, el estándar once hace referencia a las habilidades disciplinarias que debiese demostrar el profesor para conducir el aprendizaje de este eje.

Tabla 3. Estándares de matemática,

eje geometría, para egresados de carreras de pedagogía en educación básica

\begin{tabular}{|l|l|}
\hline \multicolumn{2}{|c|}{ Geometría } \\
\hline Estándar 7 & Es capaz de conducir el aprendizaje de las formas geométricas. \\
\hline Estándar 8 & Es capaz de conducir el aprendizaje de las figuras planas. \\
\hline Estándar 9 & $\begin{array}{l}\text { Está preparado para conducir el aprendizaje de conceptos y aplicaciones de la } \\
\text { medición. }\end{array}$ \\
\hline Estándar 10 & $\begin{array}{l}\text { Está preparado para conducir el aprendizaje de conceptos de perímetro, área y } \\
\text { volumen. }\end{array}$ \\
\hline Estándar 11 & Demuestra competencia disciplinaria en el eje de Geometría. \\
\hline
\end{tabular}


El eje de álgebra: este eje presenta desafíos importantes en la formación de profesores capaces de implementar esta área al currículo escolar, en especial, al traspasar de los números, que poseen un grado elevado de abstracción, a las letras que representan números. Este salto de abstracción que implica la ampliación del lenguaje en los niños y niñas constituye un desafío en la formación.

El eje de álgebra se conforma por tres estándares, como se aprecia en la tabla 4. El primero, el estándar doce se relaciona al reconocimiento de patrones y su descripción algebraica, lo que resulta de gran valor en la conceptualización del uso de variables. El siguiente estándar, el número trece, se vincula a los contenidos explícitos del currículo escolar, es decir, expresiones algebraicas, ecuaciones lineales, aplicaciones e interpretaciones de su enseñanza. Finalmente, el estándar número catorce se destina a la noción de función en general, poniendo énfasis en ecuaciones lineales, cuadráticas y sistemas de ecuaciones. Se consideran en este estándar también, la relación de orden en los números y la reflexión propia de la matemática escolar y su enseñanza en este eje.

Tabla 4. Estándares de matemática,

eje álgebra, para egresados de carreras de pedagogía en educación básica.

\begin{tabular}{|l|l|}
\hline \multicolumn{2}{|c|}{ Álgebra } \\
\hline Estándar 12 & Es capaz de conducir el aprendizaje de patrones y sucesiones. \\
\hline Estándar 13 & Está preparado para conducir el aprendizaje de expresiones algebraicas y ecuaciones. \\
\hline Estándar 14 & Demuestra competencia disciplinaria en el eje de Álgebra. \\
\hline
\end{tabular}

El eje de datos y azar: este eje se dedica al estudio de los elementos básicos de estadística descriptiva, inferencia estadística y probabilidades. El implementar de manera temprana en el currículo escolar la idea de datos y azar representa una tendencia mundial que se ve reflejada en tres líneas relevantes: primero, en la vida diaria como ciudadano, mediante la comprensión de fenómenos, noticias, estudios de frecuencia, que ayudan en la toma de decisiones. Una segunda línea es como conocimiento de base de todas las disciplinas científicas y tecnológicas que inciden en el desarrollo de la sociedad. Finalmente, una última línea lo percibe como una herramienta importante para el desarrollo del pensamiento crítico.

Para el tratamiento de este eje se han definido tres estándares, como se observa en la tabla 5. El primer estándar, el número quince se relaciona directamente con la enseñanza de la estadística, mientras que el estándar dieciséis aborda la enseñanza de la probabilidad, por último, el estándar diecisiete establece el nivel del dominio disciplinario que debe poseer el profesor en estas materias. Cabe señalar, que se entiende que la estadística y la probabilidad son ramas de la matemática que se encuentran íntimamente relacionadas, los estándares las abordan de manera separada, pues en los primeros años de escolaridad los temas de estadística se abordan de manera descriptiva y no incorporan conceptos de probabilidad. 
CONOCIMIENTO DIDÁCTICO-MATEMÁTICO PARA LA ENSEÑANZA DE LA MATEMÁTICA ELEMENTAL EN FUTUROS PROFESORES DE EDUCACIÓN BÁSICA: DISEÑO, CONSTRUCCIÓN Y VALIDACIÓN DE UN ...

Tabla 5. Estándares de matemática, eje datos y azar, para egresados de carreras de pedagogía en educación básica

\begin{tabular}{|l|l|}
\hline \multicolumn{2}{|c|}{ Datos y Azar } \\
\hline Estándar 15 & Es capaz de conducir el aprendizaje de la recolección y análisis de datos \\
\hline Estándar 16 & Está preparado para conducir el aprendizaje de las probabilidades \\
\hline Estándar 17 & Demuestra competencia disciplinaria en el eje de Datos y Azar \\
\hline
\end{tabular}

\subsection{LA MATEMÁTICA ELEMENTAL EN EL CURRÍCULO ESCOLAR CHILENO PARA LA EDUCACIÓN BÁSICA}

Para establecer la presencia otorgada a la matemática elemental en el currículo escolar chileno se analizaron las bases curriculares de matemática (Mineduc 2012). En nuestro país, las bases curriculares constituyen los aprendizajes comunes para todos los alumnos y alumnas de $1^{\circ}$ a $6^{\circ}$ año básico, organizadas mediante objetivos de aprendizaje que describen los desempeños mínimos que debiese alcanzar los estudiantes en cada asignatura y nivel educativo.

En la asignatura de matemática se busca el desarrollo del pensamiento matemático, para ello se incluyen cuatro habilidades: resolver problemas, representar, modelar, argumentar y comunicar (Mineduc 2012).

- Resolver problemas: esta habilidad busca que los estudiantes sean capaces de dar solución, a distintos tipos de situaciones problemáticas utilizando distintos tipos de estrategia, como experimentación, ensayo y error, transferencia de problemas similares ya resueltos, etc. siendo capaces de comparar los distintos caminos de solución y evaluar las respuestas obtenidas y su pertinencia.

- Representar: mediante el desarrollo de esta habilidad se espera que los estudiantes manejen una amplia variedad de representaciones matemáticas (diagramas, esquemas, gráficos, leguaje simbólico, etc.) para representar un mismo concepto, esto permitirá que los alumnos logren alcanzar un aprendizaje significativo y desarrollar la capacidad de pensar matemáticamente.

- Modelar: esta habilidad tiene por objetivo que los estudiantes construyan modelos matemáticos a partir de la identificación de patrones. Dichos modelos debiesen constituir una versión simplificada y abstracta de un sistema más complejo, que contenga los patrones y sea expresado mediante el lenguaje matemático.

- Argumentar y comunicar: con esta habilidad se busca que los estudiantes sean capaces de verbalizar y comunicar sus deducciones para luego hacer predicciones eficaces, respecto de situaciones reales. A su vez, también se busca el desarrollo de la capacidad de intuir y concluir correctamente, detectando aquellas informaciones erróneas. 
Estas habilidades cumplen un rol fundamental en la adquisición de nuevas destrezas y conceptos para lograr la resolución de problemas propios de la matemática y de otros contextos, lo que representa al foco central de la enseñanza de la matemática. Por otra parte, las bases curriculares en la asignatura de matemática organizan el aprendizaje de los estudiantes en cinco ejes temáticos, como son: Números y operaciones, Patrones y álgebra, Geometría, Medición, Datos y probabilidades.

La matemática elemental presente en el currículo escolar se organiza de acuerdo a cada uno de los ejes temáticos mencionados anteriormente, de la siguiente manera:

- Números y operaciones: este eje abarca el desarrollo del concepto de número, cálculo mental y uso de algoritmos. Luego de que los alumnos construyen y asimilan los conceptos básicos se espera que con el uso de representaciones logren aprender los algoritmos de adición, sustracción multiplicación y división, como también el sistema posicional y la estructura de los números, ampliando esto a medida que avanzan los niveles educativos para alcanzar al trabajo con números racionales.

- Patrones y álgebra: en este eje se espera que los estudiantes puedan explicar y describir relaciones de todo tipo. Los patrones, los cuales pueden ser observados a través de una secuencia de objetos, números o imágenes que representan regularidades se pueden representar en forma concreta pictórica y simbólica, se espera que los estudiantes sean capaces de sean capaces de transitar de una forma de representación a otra. Es importante considerar una base sólida en patrones, ya que esto facilitaría el desarrollo del pensamiento abstracto en las etapas educativas posteriores, hasta llegar al pensamiento algebraico.

- Geometría: los estudiantes mediante este eje deben ser capaces de reconocer, visualizar, dibujar figuras y describir propiedades y características de figuras $2 \mathrm{D}$ y 3D. Aquí se desarrollan conceptos más precisos para comprender la estructura del espacio y describir en un lenguaje más exacto lo que se conoce como entorno. También se pretende desarrollar el pensamiento espacial, mediante el estudio del movimiento de los objetos, tales como, traslación, rotación y reflexión.

- Medición: a través del desarrollo de este eje se espera que los estudiantes logren identificar características de los objetos (ancho, largo, alto, peso, volumen, etc) y cuantificarlos, para luego comparar y ordenar. Los alumnos deben ser capaces de desarrollar la habilidad para hacer mediciones, a partir de medidas no estandarizadas, para luego conocer y utilizar medidas estandarizadas, seleccionando y utilizando medidas apropiadas, según lo que se requiera medir.

- Datos y probabilidades: este eje busca responder a la necesidad de que los estudiantes sean capaces de registra, clasificar y leer información a partir de tablas y gráficos presentes en su vida cotidiana, además de introducirse en el área de la probabilidad. Todo esto se espera lograr mediante la aplicación de encuestas y formulación de preguntas basadas en la experiencia e intereses de los propios estudiantes, para luego registrar y hacer predicciones de los resultados obtenidos.

Para efectos de la investigación se han seleccionado del currículo escolar de matemática de educación básica ciertos objetivos de aprendizaje considerando los ejes temáticos tratados, de acuerdo a los primeros niveles de escolaridad, es decir, $1^{\circ}$ a $4^{\circ}$ año básico, que resultan más representativos para este estudio, como se aprecia en la tabla 6: 
CONOCIMIENTO DIDÁCTICO-MATEMÁTICO PARA LA ENSEÑANZA DE LA MATEMÁTICA ELEMENTAL EN FUTUROS PROFESORES DE EDUCACIÓN BÁSICA: DISEÑO, CONSTRUCCIÓN Y VALIDACIÓN DE UN ...

Tabla 6. Objetivos de Aprendizaje en relación al tema de las matemáticas elementales en Educación Básica (Mineduc 2012)

\begin{tabular}{|c|c|c|}
\hline Eje temático & Nivel educativo & Objetivo de aprendizaje \\
\hline $\begin{array}{l}\text { Números y } \\
\text { operaciones }\end{array}$ & $4^{\circ}$ año básico & $\begin{array}{l}\text { - Demostrar que comprende la relación entre la adición y la sus- } \\
\text { tracción al usar la "familia de operaciones" en cálculos aritméti- } \\
\text { cos y la resolución de problemas. } \\
\text { - Identificar, escribir y representar fracciones propias y los nú- } \\
\text { meros mixtos hasta el } 5 \text { de manera concreta, pictórica y simbó- } \\
\text { lica, en el contexto de la resolución de problemas. }\end{array}$ \\
\hline $\begin{array}{l}\text { Patrones y } \\
\text { Álgebra }\end{array}$ & $2^{\circ}$ ai & $\begin{array}{l}\text { - Crear, representar y continuar una variedad de patrones numé- } \\
\text { ricos y completar los elementos faltantes, de manera manual y/o } \\
\text { usando software educativo. }\end{array}$ \\
\hline Geometría & $\begin{array}{l}1^{\circ} \text { año básico } \\
2^{\circ} \text { año básico }\end{array}$ & $\begin{array}{l}\text { - Identificar en el entorno figuras 3D y figuras } 2 \mathrm{D} \text { y relacionar- } \\
\text { las, usando material concreto. } \\
\text { - Describir, comparar y construir figuras 3D (cubos, paralelepí- } \\
\text { pedos, esferas y conos) con diversos materiales. }\end{array}$ \\
\hline Medición & $4^{\circ}$ año básico & $\begin{array}{l}\text { - Medir longitudes con unidades estandarizadas ( } \mathrm{m}, \mathrm{cm} \text { ) y reali- } \\
\text { zar transformaciones entre estas unidades ( } \mathrm{m} \text { a } \mathrm{cm} \text { y viceversa) } \\
\text { en el contexto de la resolución de problemas. }\end{array}$ \\
\hline $\begin{array}{c}\text { Datos y } \\
\text { probabilidades }\end{array}$ & $3^{\circ}$ año básico & $\begin{array}{l}\text { - Registrar y ordenar datos obtenidos de juegos aleatorios con } \\
\text { dados y monedas, encontrando el menor, el mayor y estimando } \\
\text { el punto medio entre ambos. }\end{array}$ \\
\hline
\end{tabular}

\section{DISEÑO, CONSTRUCCIÓN Y VALIDACIÓN DEL CUESTIONARIO SOBRE EL CONOCIMIENTO DIDÁCTICO-MATEMÁTICO DE LA MATEMÁTICA ELEMENTAL}

Para alcanzar el propósito de construir un instrumento que permita evaluar el conocimiento didáctico-matemático para la enseñanza de las matemáticas elementales que poseen los futuros profesores de Educación Básica, nos hemos basado en el modelo propuesto por Vásquez y Alsina (Vásquez y Alsina 2015). Asimismo, se ha escogido construir un cuestionario de respuesta abierta, pues este tipo de respuesta permite obtener una mayor riqueza y detalle de las contestaciones que entreguen los futuros profesores acerca de los conocimientos didáctico-matemáticos que poseen, accediendo a aspectos que son inobservables de otra manera. Según Godino, estos conocimientos, que no siempre son accesibles por simple observación o encuesta, se pueden inferir a partir de las preguntas planteadas en el cuestionario, las que sí resultan observables (Godino 1996).

\subsection{CONSTRUCCIÓN DE LA VERSIÓN PILOTO DEL CUESTIONARIO}

Para la construcción del Cuestionario CDM-Matemáticas Elementales nos hemos apoyado en el modelo de conocimiento didáctico-matemático propuesto por Godino, el cual nos permitirá 
evaluar el conocimiento común del contenido, el conocimiento ampliado del contenido y el conocimiento especializado del contenido que poseen los futuros profesores de Educación General Básica, para enseñar matemáticas elementales (Godino 2014). La mirada de la investigación se desarrollará desde la faceta epistémica de este modelo e indagaremos en algunos aspectos esenciales de las diversas subcategorías que conforman las otras facetas.

Para la elaboración del instrumento, en una primera instancia se escoge una tarea matemática vinculada a aspectos relevantes de la matemática elemental que se desea evaluar, luego se formulan ítems relacionados con las diversas facetas del conocimiento didáctico-matemático. Desde esta perspectiva la construcción del cuestionario se orienta de manera siguiente:

\section{Selección situaciones problemáticas de enseñanza \\ 2. Selección de aspectos del contenido didáctico matemático \\ 3. Análisis de los ítems del cuestionario}

Para la selección de las situaciones problemáticas de enseñanza (ítems) se consideraron aspectos curriculares que contemplan los contenidos de la matemática elemental, los cuales tienen estricta relación con los ejes temáticos de matemática correspondientes al primer ciclo de enseñanza básica, presentes en el currículum nacional chileno (Mineduc 2012): Números y Operaciones, Patrones y Álgebra, Geometría, Medición, Datos y Probabilidades.

Las situaciones problemáticas de enseñanza que se proponen en el cuestionario fueron tomadas desde los estándares orientadores para egresados de carreras de pedagogía en educación básica, disciplinarios de matemática. A partir de esta selección, las situaciones fueron adaptadas y reestructuradas para incorporar aspectos parciales de las categorías y subcategorías que componen el modelo de conocimiento didáctico-matemático. Cada situación problemática de enseñanza presente en el instrumento sitúa al futuro profesor desde un escenario hipotético, con ello se espera que sea capaz de utilizar todas las herramientas entregadas durante su formación inicial y busque dar respuesta tanto a las tareas matemáticas existentes detrás de cada ítem del cuestionario, como también a los aspectos del conocimiento didáctico-matemático.

Para seleccionar los aspectos del contenido didáctico-matemático, se consideraron aspectos parciales de las categorías y subcategorías que componen el conocimiento común, ampliado y especializado a partir de las facetas involucradas en dicho modelo. Desde esta perspectiva, y por medio de las situaciones problemáticas del cuestionario, se formularon preguntas (subítems) que permiten evaluar las categorías y subcategorías del conocimiento didáctico-matemático, tales como: resuelva el problema planteado, para evaluar el conocimiento común del contenido en relación con la matemática elemental; ¿Con qué conceptos más avanzados del currículo escolar relaciona el contenido involucrado en la resolución de problema planteado?, para evaluar el conocimiento ampliado del contenido en relación con la matemáticas elementales; ¿qué conceptos y/o propiedades matemáticas deben usar los alumnos para dar una solución correcta?, para evaluar el conocimiento especializado; describa las posibles dificultades presentes en las respuestas incorrectas, que han llevado al alumno a responder de manera errónea, para evaluar el conocimiento del contenido en relación con los estudiantes; ¿qué estrategias utilizaría usted como profesor para orientar a aquellos alumnos que no han sabido resolver el problema?, para evaluar el conocimiento del contenido en relación con la enseñanza; ¿para qué curso considera usted 
pertinente el problema planteado de acuerdo con el currículo nacional?, para evaluar el conocimiento del contenido en relación con el currículo.

\subsection{REVISIÓN DEL INSTRUMENTO MEDIANTE JUICIO DE EXPERTOS Y APLICACIÓN PILOTO}

La revisión del instrumento contempla un proceso de validación relacionado con el estudio de los contenidos vinculados a la matemática elemental, basados en los ejes temáticos propuestos por el ministerio de educación para primer ciclo de enseñanza básica (Mineduc 2012), como así también, la fiabilidad de los ítems. A partir de esto se consideraron dos etapas: juicio de expertos y análisis de los ítems a partir de la aplicación piloto del cuestionario.

\subsubsection{Juicio de expertos}

El instrumento fue sometido a un proceso de validación por tres expertos en didáctica de la matemática y formación de profesores en Chile. Esto permitió analizar la pertinencia de los ítems del cuestionario según las categorías del modelo de conocimiento didácticomatemático.

Los expertos recibieron vía correo electrónico el instrumento y una pauta para evaluar los siguientes aspectos de los ítems del cuestionario:

Grado de correspondencia: señalan si las preguntas realizadas miden la categoría y/o subcategoría del modelo de conocimiento didáctico matemático.

Formulación de las preguntas: Redacción y comprensión de los enunciados.

A partir de los comentarios y sugerencias recibidos por parte de los expertos, se reformularon y afinaron distintos aspectos de los ítems del cuestionario, tales como redacción, reformulación de preguntas, incorporación y sustitución de nuevos ítems, de la manera siguiente:

Ítem 1: se mejoró la redacción de los subítems 1a) y 1c) y se agregó el subítem 1e) referente al conocimiento especializado del contenido, subcategoría conocimiento en relación con el currículo.

Ítem 2: se reformuló los subítems 2a) y 2b) y se incorporó el subítem 2e) relacionado con el conocimiento especializado del contenido, subcategoría conocimiento en relación con la enseñanza.

Ítems 3 y 5: no se recibieron observaciones por parte de los evaluadores, por lo que no se realizaron modificaciones.

Ítem 4: se reestructuró el subítem 4b), como dos preguntas independientes, dando origen a los nuevos subítems 4b) y 4c) que evalúan el conocimiento especializado del contenido, subcategoría conocimiento en relación con la enseñanza. El subítem 4c) que existía anteriormente ha quedado estipulado como 4d). 
Estudios Pedagógicos XLIV, $\mathrm{N}^{\circ}$ 1: 25-48, 2018

CONOCIMIENTO DIDÁCTICO-MATEMÁTICO PARA LA ENSEÑANZA DE LA MATEMÁTICA ELEMENTAL EN FUTUROS PROFESORES DE EDUCACIÓN BÁSICA: DISEÑO, CONSTRUCCIÓN Y VALIDACIÓN DE UN ...

Ítems 6 y 8: dos de los evaluadores consideran pertinente suprimir los ítems, dando prioridad a los otros ítems pues resultan más enriquecedores en cuanto a la medición de las categorías y subcategorías del modelo de conocimiento didáctico-matemático.

Ítem 7: se agregó un apartado 7c) que evalúe el conocimiento ampliado del contenido, de esta forma surgió la pregunta: “¿Con qué conceptos más avanzados del currículo escolar relaciona el contenido involucrado en la resolución de este problema?”

En su versión inicial (Anexo 1), el cuestionario se encontraba compuesto por 8 ítems de respuesta abierta, una vez sometido al juicio de expertos, este quedo constituido en su versión final por 6 ítems de respuesta abierta, los cuales permiten abordar las categorías globales y subcategorías del modelo de conocimiento didáctico-matemático (Godino 2014).

\subsubsection{Aplicación piloto del cuestionario}

Luego de la reestructuración del cuestionario a partir del juicio de expertos, se llevó a cabo la aplicación piloto del instrumento a 12 estudiantes de pedagogía en Educación General Básica que cursaban su cuarto año de carrera. El objetivo de esto es analizar las posibles limitaciones que podría presentar el instrumento en cuanto a su redacción, orden, comprensión de los ítems y subítems diseñados, como así también la pertinencia del tiempo para responder (90 minutos), otorgando la validación del instrumento.

A partir de las consultas y sugerencias planteadas durante la aplicación piloto del cuestionario por parte de los futuros profesores y sus respuestas plasmadas en el mismo, surgieron los siguientes cambios al instrumento:

Se mejoró la redacción de los ítems 1) y 5), a su vez, se eliminó el subítem 2b), pues coincide su respuesta con la respuesta del subítem $2 a$ ). Por otra parte, se ha fusionado el subítem 4a) y 4b), pues la pregunta 4b) tiene relación con las actividades que se propondrían para la enseñanza del contenido, con lo cual se da respuesta a esta pregunta en la pregunta 4a) al mencionar las estrategias de enseñanza. De este modo, el subítem 4b) es reemplazado por una nueva pregunta.

En cuanto al tiempo estimado para responder el cuestionario, que consistía en 90 minutos, se considera pertinente, pues todos los estudiantes entregaron sus respuestas en el tiempo establecido.

En la revisión del instrumento mediante la aplicación piloto también se consideró el análisis de las respuestas del cuestionario. Para ello se corrigió el cuestionario a partir de una rúbrica, asignado puntaje a las respuestas obtenidas, en este caso, 2 puntos si la respuesta es correcta, 1 punto si la respuesta es parcialmente correcta y 0 si la respuesta es incorrecta. De acuerdo a las puntuaciones establecidas en los ítems del cuestionario, el puntaje máximo es de 44 puntos y el mínimo 0 puntos.

A partir de los resultados obtenidos, no se observaron puntuaciones máximas y los puntajes totales variaron entre 8 y 36 puntos. El porcentaje de logro alcanzado por los futuros profesore es del $57 \%$, considerando la media obtenida de 20,5 puntos. Con respecto al índice de dificultad (ID), el cual valora la dificultad que implica la resolución de las situaciones problemáticas de enseñanza que propone el instrumento. Según Muñiz, lo define como la razón entre el número de aciertos/número de respuestas (Muñiz 1994). El ID toma valores entre 0 y 1 , siendo el 0 quien indica un alto grado de dificultad, y 1 un 
grado de máxima facilidad, en este sentido, los índices de dificultad media son los que mejor discriminan. Para calcular dicho índice se clasificaron las respuestas en correctas e incorrectas, no se consideraron aquellas respuestas no respondidas, de este modo fue posible visualizar que situaciones problemáticas resultaron más sencillas o complejas de responder para este grupo de futuros profesores. La tabla 7 muestra un resumen estadístico de los datos:

Tabla 7: Índice de dificultad de los ítems del cuestionario

\begin{tabular}{|c|c|c|c|c|c|c|c|c|c|c|c|c|c|c|c|c|c|c|c|c|c|}
\hline \multirow{2}{*}{ Ítem } & \multicolumn{5}{|c|}{1} & \multicolumn{4}{|c|}{2} & \multicolumn{2}{|c|}{3} & \multicolumn{4}{|c|}{4} & \multicolumn{3}{|c|}{5} & \multicolumn{3}{|c|}{6} \\
\hline & a) & b) & c) & d) & e) & a) & b) & c) & d) & a) & b) & a) & b) & c) & d) & a) & b) & c) & a) & b) & c) \\
\hline ID $(\%)$ & 95 & 74 & 50 & 62 & 66 & 98 & 66 & 88 & 66 & 47 & 62 & 62 & 70 & 62 & 66 & 70 & 70 & 66 & 66 & 62 & 0 \\
\hline
\end{tabular}

El cuestionario presentó una dificultad media de un 63\%, como se muestra en la tabla 6. El ítem del cuestionario de mayor dificultad corresponde al 6c), relacionados con el conocimiento ampliado del contenido sobre el eje de medición. Los ítems con menor dificultad fueron 1a), 2a) y 2c), los dos primeros se vinculan al conocimiento común del contenido sobre los ejes temáticos de números y operaciones - patrones y álgebra, respectivamente, mientras que el tercer ítem se relaciona con el conocimiento especializado del contenido en relación con el currículo.

A continuación se analizan las respuestas obtenidas de acuerdo a los ejes temáticos presentes en los ítems del cuestionario, vale decir, números y operaciones, patrones y álgebra, geometría, medición, datos y probabilidades, describiendo los principales resultados y hallazgos. Por otra parte, se ha estudiado el tipo de conocimiento didáctico matemático presente en cada eje temático, como lo es el conocimiento común del contenido, el conocimiento ampliado del contenido y el conocimiento especializado del contenido.

\section{a) Eje temático: Números y Operaciones}

Los ítems del cuestionario que comprenden el eje de números y operaciones corresponden al ítem 1 y 3, los cuales se encuentran tomados y adaptados a partir de la propuesta otorgada por los estándares orientadores para egresados de carreras de pedagogía en educación básica, disciplinarios de matemática con ejemplos.

El objetivo de estos ítems es evaluar el conocimiento común del contenido y conocimiento especializado del contenido, en relación con los estudiantes, enseñanza y el currículo, vinculados a la adición de fracciones utilizando esquemas adecuados que permitan representar la operatoria y la resolución de problemas en situaciones aditivas.

En la tabla 8 se observa que hubo un alto porcentaje de respuestas correctas. El 83,3\% de los futuros profesores logra resolver la situación planteada: identificar la representación de adición de fracciones, abordando el conocimiento común del contenido. 
Estudios Pedagógicos XLIV, $\mathrm{N}^{\circ}$ 1: 25-48, 2018

CONOCIMIENTO DIDÁCTICO-MATEMÁTICO PARA LA ENSEÑANZA DE LA MATEMÁTICA ELEMENTAL EN FUTUROS PROFESORES DE EDUCACIÓN BÁSICA: DISEÑO, CONSTRUCCIÓN Y VALIDACIÓN DE UN ...

Tabla 8. Frecuencia de respuestas al subítem 1a) $(\mathrm{n}=12)$

\begin{tabular}{|c|c|c|c|c|c|c|c|c|}
\hline \multicolumn{8}{|c|}{ Conocimiento Común del Contenido } \\
\hline $\begin{array}{c}\text { Eje temático: Números } \\
\text { y Operaciones }\end{array}$ & $\begin{array}{c}\text { Respuesta } \\
\text { correcta }\end{array}$ & \multicolumn{2}{|c|}{$\begin{array}{c}\text { Respuesta } \\
\text { parcialmente correcta }\end{array}$} & $\begin{array}{c}\text { Respuesta } \\
\text { incorrecta }\end{array}$ & \multicolumn{2}{c|}{$\begin{array}{c}\text { No } \\
\text { responde }\end{array}$} \\
\hline Subítem & Frecuencia & $\%$ & Frecuencia & $\%$ & Frecuencia & $\%$ & Frecuencia & $\%$ \\
\hline 1a & 10 & 83,3 & 2 & 16,7 & 0 & 0 & 0 & 0 \\
\hline
\end{tabular}

Al analizar las respuestas del cuestionario, en el subítem 1a), 2 futuros profesores no logran obtener una respuesta totalmente correcta, los errores cometidos tienen relación con la representación independiente de cada fracción.

En cuanto al conocimiento especializado del contenido (tabla 9) se observa una mayor cantidad de respuestas correctas en relación al conocimiento del contenido especializado, donde los futuros profesores identifican los conceptos matemáticos que se requieren para dar solución al problema, por ejemplo, "se necesita conocer el concepto de amplificación y manejar la representación de fracciones de manera pictórica" (futuro profesor 3). Así también, el conocimiento en relación con el currículo, donde el 50\% de los participantes logra identificar el nivel y curso para el cual es pertinente el problema.

Tabla 9. Frecuencia de respuestas a los subítems 1b), c), d), e) y 3a), 3b) (n=12)

\begin{tabular}{|c|c|c|c|c|c|c|c|c|c|}
\hline \multicolumn{8}{|c|}{ Conocimiento Especializado del Contenido } \\
\hline $\begin{array}{c}\text { Eje temático: Números } \\
\text { y Operaciones }\end{array}$ & $\begin{array}{c}\text { Respuesta } \\
\text { correcta }\end{array}$ & \multicolumn{2}{|c|}{$\begin{array}{c}\text { Respuesta } \\
\text { parcialmente correcta }\end{array}$} & \multicolumn{2}{c|}{$\begin{array}{c}\text { Respuesta } \\
\text { incorrecta }\end{array}$} & \multicolumn{2}{c|}{$\begin{array}{c}\text { No } \\
\text { responde }\end{array}$} \\
\hline Subítem & Frecuencia & $\%$ & Frecuencia & $\%$ & Frecuencia & $\%$ & Frecuencia & $\%$ \\
\hline $1 \mathrm{~b}$ & 7 & 58,4 & 4 & 33,3 & 1 & 8,3 & 0 & 0 \\
\hline $1 \mathrm{c}$ & 5 & 41,7 & 4 & 33,3 & 2 & 16,7 & 1 & 8,3 \\
\hline $1 \mathrm{~d}$ & 5 & 41,7 & 6 & 50 & 1 & 8,3 & 0 & 0 \\
\hline $1 \mathrm{e}$ & 6 & 50 & 4 & 33,3 & 2 & 16,7 & 0 & 0 \\
\hline $3 \mathrm{a}$ & 4 & 33,3 & 5 & 41,7 & 3 & 25 & 0 & 0 \\
\hline $3 \mathrm{~b}$ & 5 & 41,7 & 6 & 50 & 0 & 0 & 1 & 8,3 \\
\hline
\end{tabular}

Los subítems que tuvieron menor cantidad de respuestas correctas están relacionados con el conocimiento del contenido en relación con la enseñanza y los estudiantes. Los errores que se observan en las respuestas plasmados por los futuros profesores se relacionan con la 
capacidad de identificar en su totalidad las dificultades presentes en las respuestas incorrectas que se entregaron como solución a las situaciones planteadas y señalar estrategias de mejora para ellas, por ejemplo, el disponer de estrategias para enseñar la adición y sustracción de fracciones, y utilizar esquemas adecuados que permiten representar estas operaciones.

\section{b) Eje temático: Patrones y Álgebra}

Este ítem se ha tomado y adaptados a partir de la propuesta otorgada por los estándares orientadores para egresados de carreras de pedagogía en educación básica, disciplinarios de matemática con ejemplos. Su propósito es evaluar el conocimiento común del contenido, conocimiento del contenido en relación con los estudiantes, enseñanza y currículo desde la descripción de patrones y secuencias.

En este ítem los futuros profesores logran un buen desempeño, en especial al identificar la figura observando el patrón y siguiendo la secuencia numérica lo que evidencia un buen manejo en el conocimiento común del contenido (tabla 10).

Tabla 10. Frecuencia de respuestas al subítem 2a) $(\mathrm{n}=12)$

\begin{tabular}{|c|c|c|c|c|c|c|c|c|}
\hline \multicolumn{8}{|c|}{ Conocimiento Común del Contenido } \\
\hline $\begin{array}{c}\text { Eje temático: } \\
\text { Patrones y Álgebra }\end{array}$ & $\begin{array}{c}\text { Respuesta } \\
\text { correcta }\end{array}$ & \multicolumn{2}{c|}{$\begin{array}{c}\text { Respuesta } \\
\text { parcialmente correcta }\end{array}$} & \multicolumn{2}{c|}{$\begin{array}{c}\text { Respuesta } \\
\text { incorrecta }\end{array}$} & \multicolumn{2}{c|}{$\begin{array}{c}\text { No } \\
\text { responde }\end{array}$} \\
\hline Subítem & Frecuencia & $\%$ & Frecuencia & $\%$ & Frecuencia & $\%$ & Frecuencia & $\%$ \\
\hline 2a & 11 & 91,7 & 1 & 8,3 & 0 & 0 & 0 & 0 \\
\hline
\end{tabular}

Alrededor de la mitad de los participantes responde correctamente las preguntas relacionadas con el conocimiento especializado del contenido (tabla 11), destacando el conocimiento en relación con el currículo en el subítem 2c).

Tabla 11. Frecuencia de respuestas a los subítems 2b), 2c) y 2d) (n = 12)

\begin{tabular}{|c|c|c|c|c|c|c|c|c|}
\hline \multicolumn{7}{|c|}{ Conocimiento Especializado del Contenido } \\
\hline $\begin{array}{c}\text { Eje temático: } \\
\text { Patrones y Álgebra }\end{array}$ & \multicolumn{2}{|c|}{$\begin{array}{c}\text { Respuesta } \\
\text { correcta }\end{array}$} & $\begin{array}{c}\text { Respuesta } \\
\text { parcialmente } \\
\text { correcta }\end{array}$ & $\begin{array}{c}\text { Respuesta } \\
\text { incorrecta }\end{array}$ & \multicolumn{2}{c|}{$\begin{array}{c}\text { No } \\
\text { responde }\end{array}$} \\
\hline Subítem & Frecuencia & $\%$ & Frecuencia & $\%$ & Frecuencia & $\%$ & Frecuencia & $\%$ \\
\hline $2 \mathrm{~b}$ & 6 & 50 & 3 & 25 & 3 & 25 & 0 & 0 \\
\hline $2 \mathrm{c}$ & 9 & 75 & 3 & 25 & 0 & 0 & 0 & 0 \\
\hline $2 \mathrm{~d}$ & 6 & 50 & 3 & 25 & 3 & 25 & 0 & 0 \\
\hline
\end{tabular}


Estudios Pedagógicos XLIV, $\mathrm{N}^{\circ}$ 1: 25-48, 2018

CONOCIMIENTO DIDÁCTICO-MATEMÁTICO PARA LA ENSEÑANZA DE LA MATEMÁTICA ELEMENTAL EN FUTUROS PROFESORES DE EDUCACIÓN BÁSICA: DISEÑO, CONSTRUCCIÓN Y VALIDACIÓN DE UN ...

Las mayores dificultades se presentan en el subítem 2b) sobre conocimiento en relación con los estudiantes, en el cual el futuro profesor debe describir los problemas que tuvieron los estudiantes para determinar el patrón de la figura que falta en la secuencia. Del mismo modo, el subítem 2d) relacionado al conocimiento en relación con la enseñanza, presenta dificultades, los futuros profesores plantean "utilizar material concreto" (futuro profesor 7) como un buen recurso para representar el problema, pero no logran identificar el tipo de material, ni justificar su elección.

\section{c) Eje temático: Geometría}

Este ítem se ha tomado y adaptados a partir de la propuesta otorgada por los estándares orientadores para egresados de carreras de pedagogía en educación básica, disciplinarios de matemática con ejemplos. Su objetivo es evaluar el conocimiento especializado del contenido en relación con la enseñanza y el currículo, mediante el reconocimiento y descripción de los cuerpos geométricos.

Se observa un mejor desempeño en el conocimiento en relación con el currículo (tabla 12), un $66,6 \%$ de los futuros profesores logra identificar el nivel y curso para el cual es pertinente la situación problemática planteada.

Tabla 12. Frecuencia de respuestas a los subítems 4a), 4b), 4c) y 4d) (n=12)

\begin{tabular}{|c|c|c|c|c|c|c|c|c|}
\hline \multicolumn{7}{|c|}{ Conocimiento Especializado del Contenido } \\
\hline $\begin{array}{c}\text { Eje temático: } \\
\text { Geometría }\end{array}$ & $\begin{array}{c}\text { Respuesta } \\
\text { correcta }\end{array}$ & \multicolumn{2}{|c|}{$\begin{array}{c}\text { Respuesta } \\
\text { parcialmente correcta }\end{array}$} & \multicolumn{2}{|c|}{$\begin{array}{c}\text { Respuesta } \\
\text { incorrecta }\end{array}$} & \multicolumn{3}{c|}{$\begin{array}{c}\text { No } \\
\text { responde }\end{array}$} \\
\hline Subítem & Frecuencia & $\%$ & Frecuencia & $\%$ & Frecuencia & $\%$ & Frecuencia & $\%$ \\
\hline $4 \mathrm{a}$ & 5 & 41,7 & 6 & 50 & 1 & 8,3 & 0 & 0 \\
\hline $4 \mathrm{~b}$ & 8 & 66,6 & 2 & 16,7 & 2 & 16,7 & 0 & 0 \\
\hline $4 \mathrm{c}$ & 5 & 41,7 & 6 & 50 & 1 & 8,3 & 0 & 0 \\
\hline $4 \mathrm{~d}$ & 6 & 50 & 4 & 33,3 & 1 & 8,3 & 1 & 8,3 \\
\hline
\end{tabular}

Al evaluar el conocimiento especializado del contenido en relación con la enseñanza, podemos observar que menos de la mitad de los participantes responden de manera correcta a cada subítem presentado. Al analizar las respuestas del cuestionario se observa que los futuros profesores presentan mayor dificultad al plantear preguntas a sus estudiantes en relación al reconocimiento de formas geométricas de su entorno y su descripción mediante lenguaje geométrico básico, como también, en la evaluación del contenido propuesto. En las respuestas señaladas se destacan aspectos importantes de este eje temático, en torno al contenido como lo es la clasificación de los cuerpos geométricos y sus características. 


\section{d) Eje temático: Datos y Probabilidades}

El ítem ha sido tomado y adaptado a partir de los estándares orientadores para egresados de carreras de pedagogía en educación básica, disciplinarios de matemática con ejemplos. El propósito de este ítem es evaluar el conocimiento común del contenido y conocimiento especializado, en relación con el currículo y conocimiento del contenido especializado, a través, del cálculo de la media aritmética y su interpretación.

Como se observa en la tabla 13, el conocimiento común del contenido de los futuros profesores sobre la capacidad de relacionar diversas representaciones y metáforas con el concepto de media aritmética de un conjunto de datos está presente en un 66,6\%.

Tabla 13. Frecuencia de respuestas al subítem 5a) $(\mathrm{n}=12)$

\begin{tabular}{|c|c|c|c|c|c|c|c|c|}
\hline \multicolumn{8}{|c|}{ Conocimiento Común del Contenido } \\
\hline $\begin{array}{c}\text { Eje temático: Datos } \\
\text { y Probabilidades }\end{array}$ & $\begin{array}{c}\text { Respuesta } \\
\text { correcta }\end{array}$ & $\begin{array}{c}\text { Respuesta } \\
\text { parcialmente correcta }\end{array}$ & \multicolumn{2}{c|}{$\begin{array}{c}\text { Respuesta } \\
\text { incorrecta }\end{array}$} & \multicolumn{2}{c|}{$\begin{array}{c}\text { No } \\
\text { responde }\end{array}$} \\
\hline Subítem & Frecuencia & $\%$ & Frecuencia & $\%$ & Frecuencia & $\%$ & Frecuencia & $\%$ \\
\hline 5a & 8 & 66,6 & 2 & 16,7 & 2 & 16,7 & 0 & 0 \\
\hline
\end{tabular}

En el conocimiento especializado del contenido (tabla 14), destaca el conocimiento en relación con el currículo, en el subítem 5b) con un 66,6\% de respuestas correctas, en estos casos los futuros profesores logran identificar el nivel para el cual corresponde esta situación.

Tabla 14. Frecuencia de respuestas a los subítems 5b) y 5c) $(\mathrm{n}=12)$

\begin{tabular}{|c|c|c|c|c|c|c|c|c|}
\hline \multicolumn{7}{|c|}{ Conocimiento Especializado del Contenido } \\
\hline $\begin{array}{c}\text { Eje temático: Datos } \\
\text { y probabilidades }\end{array}$ & $\begin{array}{c}\text { Respuesta } \\
\text { correcta }\end{array}$ & $\begin{array}{c}\text { Respuesta } \\
\text { parcialmente correcta }\end{array}$ & \multicolumn{2}{|c|}{$\begin{array}{c}\text { Respuesta } \\
\text { incorrecta }\end{array}$} & \multicolumn{3}{c|}{$\begin{array}{c}\text { No } \\
\text { responde }\end{array}$} \\
\hline Subítem & Frecuencia & $\%$ & Frecuencia & $\%$ & Frecuencia & $\%$ & Frecuencia & $\%$ \\
\hline $5 b$ & 8 & 66,6 & 2 & 16,7 & 0 & 0 & 2 & 16,7 \\
\hline $5 \mathrm{c}$ & 6 & 50 & 5 & 41,7 & 0 & & 1 & 8.3 \\
\hline
\end{tabular}

Mientras que el subítem 5c) tiene relación con el conocimiento del contenido especializado sólo alcanza un 50\% de aprobación, la mitad de los futuros profesores no logra identificar las limitaciones para calcular la media aritmética, mediante el procedimiento que entrega la situación problemática. 
Estudios Pedagógicos XLIV, $\mathrm{N}^{\circ}$ 1: 25-48, 2018

CONOCIMIENTO DIDÁCTICO-MATEMÁTICO PARA LA ENSEÑANZA DE LA MATEMÁTICA ELEMENTAL EN FUTUROS PROFESORES DE EDUCACIÓN BÁSICA: DISEÑO, CONSTRUCCIÓN Y VALIDACIÓN DE UN ...

\section{e) Eje temático: Medición}

Este ítem se encuentra tomado y adaptado a partir de la propuesta otorgada por los estándares orientadores para egresados de carreras de pedagogía en educación básica, disciplinarios de matemática con ejemplos. Su objetivo es evaluar el conocimiento especializado, en relación con la enseñanza y el conocimiento ampliado del contenido, mediante el uso de estrategias basadas en la visualización, utilizados por alumnos y alumnas para predecir o estimar.

La tabla 15 muestra que los futuros profesores presentaron dificultades para generar actividades que permitieran responder la pregunta planteada en la situación problemática y también estrategias para controlar los errores que cometen los alumnos al medir sin el uso de instrumentos graduados, revelando una debilidad en el conocimiento especializado del contenido, en relación con la enseñanza.

Tabla 15. Frecuencia de respuestas a los subítems 6a) y 6d) $(\mathrm{n}=12)$

\begin{tabular}{|c|c|c|c|c|c|c|c|c|}
\hline \multicolumn{8}{|c|}{ Conocimiento Especializado del Contenido } \\
\hline $\begin{array}{c}\text { Eje Temático: } \\
\text { Medición }\end{array}$ & $\begin{array}{c}\text { Respuesta } \\
\text { correcta }\end{array}$ & $\begin{array}{c}\text { Respuesta } \\
\text { parcialmente correcta }\end{array}$ & \multicolumn{2}{c|}{$\begin{array}{c}\text { Respuesta } \\
\text { incorrecta }\end{array}$} & \multicolumn{2}{c|}{$\begin{array}{c}\text { No } \\
\text { responde }\end{array}$} \\
\hline Subítem & Frecuencia & $\%$ & Frecuencia & $\%$ & Frecuencia & $\%$ & Frecuencia & $\%$ \\
\hline $6 \mathrm{a}$ & 6 & 50 & 4 & 33,3 & 2 & 16,7 & 0 & 0 \\
\hline 6b & 5 & 41,7 & 6 & 50 & 1 & 8,3 & 0 & 0 \\
\hline
\end{tabular}

En cuanto al conocimiento ampliado del contenido (tabla 16) no se observa un buen desempeño por parte de los futuros profesores, no logran relacionar el concepto de estimación, con otros conceptos más avanzados del currículum escolar.

Tabla 16. Frecuencia de respuestas al subítem 6c) $(\mathrm{n}=12)$

\begin{tabular}{|c|c|c|c|c|c|c|c|c|}
\hline \multicolumn{7}{|c|}{ Conocimiento Ampliado del Contenido } \\
\hline $\begin{array}{c}\text { Eje Temático: } \\
\text { Medición }\end{array}$ & $\begin{array}{c}\text { Respuesta } \\
\text { correcta }\end{array}$ & \multicolumn{2}{c|}{$\begin{array}{c}\text { Respuesta } \\
\text { parcialmente correcta }\end{array}$} & \multicolumn{2}{c|}{$\begin{array}{c}\text { Respuesta } \\
\text { incorrecta }\end{array}$} & \multicolumn{2}{c|}{$\begin{array}{c}\text { No } \\
\text { responde }\end{array}$} \\
\hline Subítem & Frecuencia & $\%$ & Frecuencia & $\%$ & Frecuencia & $\%$ & Frecuencia & $\%$ \\
\hline $6 c$ & 0 & 0 & 3 & 25 & 6 & 75 & 3 & 25 \\
\hline
\end{tabular}

Este análisis dio lugar a la versión final del cuestionario CDM - Matemáticas Elementales, quedando conformado por seis ítems de respuesta abierta (Anexo 2). 

FUTUROS PROFESORES DE EDUCACIÓN BÁSICA: DISEÑO, CONSTRUCCIÓN Y VALIDACIÓN DE UN ...

\section{CONSIDERACIONES FINALES}

El desarrollo de este estudio ha permitido observar la necesidad de contar con un instrumento de evaluación que describa el conocimiento didáctico-matemático que poseen los futuros profesores de Educación Básica para enseñar matemáticas elementales.

Mediante este trabajo, se ha mostrado el proceso de diseño, construcción y validación del instrumento, destacando la valoración del juicio de expertos y el análisis de la aplicación piloto del instrumento, lo que ha llevado a refinar y elaborar la versión final del cuestionario CDM-Matemáticas Elementales.

El analizar el conocimiento didáctico-matemático que poseen los futuros profesores de Educación General Básica para enseñar matemáticas elementales, permitió evaluar aspectos parciales en el desarrollo del conocimiento común, ampliado y especializado del contenido.

Por otra parte, a partir de la aplicación piloto del instrumento se ha observado, de manera parcial, el buen nivel que manejan los futuros profesores en cuanto al conocimiento común del contenido, destacando el manejo de los contenidos para abordar situaciones en relación a los ejes de números y operaciones, álgebra y patrones numéricos, datos y probabilidades; el nivel intermedio en el conocimiento especializado del contenido, destacando el conocimiento en relación con el currículo, vinculado a la identificación de los niveles educativos en que se desarrolla una tarea matemática relacionada con los ejes de números y operaciones, geometría y dejando en evidencia el aporte que se debe seguir realizado en la formación de profesores hacia la adquisición del conocimiento en relación con la enseñanza y los estudiantes; y por último las deficiencias que poseen los futuros profesores en relación al conocimiento ampliado del contenido, al no manejar conocimientos más avanzados del currículum escolar relacionados con el eje de medición. Siendo este último eje, uno de los más débiles en cuanto a los contenidos matemáticos presentes en el cuestionario.

Finalmente, a partir de los resultados obtenidos se ha logrado un primer acercamiento, a través de los conocimientos plasmados en las respuestas obtenidas del cuestionario, al conocimiento didáctico-matemático sobre la matemática elemental. A partir de estos resultados aún parciales queda en manifiesto el desafío que enfrenta la formación del profesorado en las distintas áreas del conocimiento matemático que deben desarrollar los futuros profesores a lo largo de su carrera profesional en los diversos niveles educativos en que se desempeñen.

\section{REFERENCIAS BIBLIOGRÁFICAS}

Godino, J. D. (1996). Mathematical concepts, their meaning and understanding. In: Puig, L.; Gutierrez, A. (Ed.). Proceedings of the XX PME Conference. Valencia: Universidad de Valencia, 417- 424.

Godino, J. D. (2002). Un enfoque ontológico y semiótico de la cognición matemática. Recherches en Didactique des Mathématiques, 22(2/3), 237-284.

Godino, J. D. (2009). Categorías de análisis de los conocimientos del profesor de matemática. UNION, Revista Iberoamericana de Educación Matemática, 20, 13-31

Godino, J. D. (2014). Síntesis del enfoque ontosemiótico del conocimiento y la instrucción matemática: motivación, supuestos y herramientas teóricas. Universidad de Granada. http:// www.ugr.es/local/jgodino/eos/sintesis_EOS_24agosto14.pdf 
Godino, J. D. Batanero, C. y Font, V. (2007). The onto-semiotic approach to research in mathematics education. ZDM. The International Journal on Mathematics Education, 39 (1-2), 127-135.

Godino, J.D. y Pino-Fan, L. (2013). The mathematical knowledge for teaching. A view from onto-semiotic approach to mathematical knowledge and instruction. In: Ubuz, B.; Haser, Ç.; Mariotti, M. (Ed.) Proceedings of the Eighth Congress of the European Society for Research in Mathematics Education (3325-3336). Antalya, Turkey.

Ma, H. C., Ball, D. L. y Shilling, S. G. (2008). Unpacking pedagogical content knowledge: Conceptualizing and measuring teachers' topic-specific knowledge of student. Journal for Research in Mathematics Education, 39, 372-400.

Lewin, R., et al. Números. ReFIP Matemática. Recursos para la Formación Inicial de Profesores de Educación Básica. Santiago, Chile, Ediciones SM, 2014.

Ma, L. (1999). Knowing and teaching elementary mathematics: Teachers' understanding of fundamental mathematics in China and the United States. Mahwah, N.J.: Lawrence Erlbaum Associates.

Martínez, S., Varas, M. L. Álgebra. ReFIP Matemática. Recursos para la Formación Inicial de Profesores de Educación Básica. Santiago, Chile, Ediciones SM, 2014.

Ministerio de Educación (2012). Bases Curriculares 2012: Educación Básica Matemática. Unidad de currículo y Evaluación: Santiago de Chile, 2012

Ministerio de Educación (2012). Estándares Orientadores para la formación Inicial Docente. Unidad de Curriculum y Evaluación. Santiago de Chile, 2012

Ministerio de Educación, (2015). Resultados INICIA 2014.Santiago, Chile

Muñiz, J. (1994). Teoría clásica de los test. Madrid: Pirámide.

Schoenfeld, A. H. y Kilpatrick, J. (2008). Towards a theory of profiency in teaching mathematics. En D. Tirosh \& T. Wood (eds.), Tools and Processes in Mathematics Teacher Education (321-354). Rottermdam: Sense Publishers.

Tatto, M.; J. Schwille; L. Ingvarson; G. Rowley y R. Peck. (2012). Policy, Practice, and Readiness to Teach Primary and Secondary Mathematics in 17 countries: Findings from the IEA Teacher Education and Development Study in Mathematics (TEDS-M).

Ministerio de Educación (2012). Estándares Orientadores para la formación Inicial Docente. Estándares disciplinarios de matemática con ejemplos. Unidad de Curriculum y Evaluación. Santiago de Chile.

Pino-Fan, L. R., Godino, J. D., y Font, V. (2013). Diseño y aplicación de un instrumento para explorar la faceta epistémica del conocimiento didáctico-matemático de futuros profesores sobre la derivada (2 $2^{\mathrm{a}}$ parte). Revemat: Revista Eletrônica de Educação Matemática, 8, 1-47.

Vásquez, C. y Alsina, A. (2015). Conocimiento Didáctico-Matemático del Profesorado de Educación Primaria sobre Probabilidad: Diseño, Construcción y Validación de un Instrumento de Evaluación. Bolema, v. 29, n. 52, p. 681-703. 
Estudios Pedagógicos XLIV, № 1: 25-48, 2018

CONOCIMIENTO DIDÁCTICO-MATEMÁTICO PARA LA ENSEÑANZA DE LA MATEMÁTICA ELEMENTAL EN FUTUROS PROFESORES DE EDUCACIÓN BÁSICA: DISEÑO, CONSTRUCCIÓN Y VALIDACIÓN DE UN ...

\section{ANEXO 1: ÍTEMS VERSIÓN INICIAL CUESTIONARIO CDM-MATEMÁTICAS ELEMENTALES}

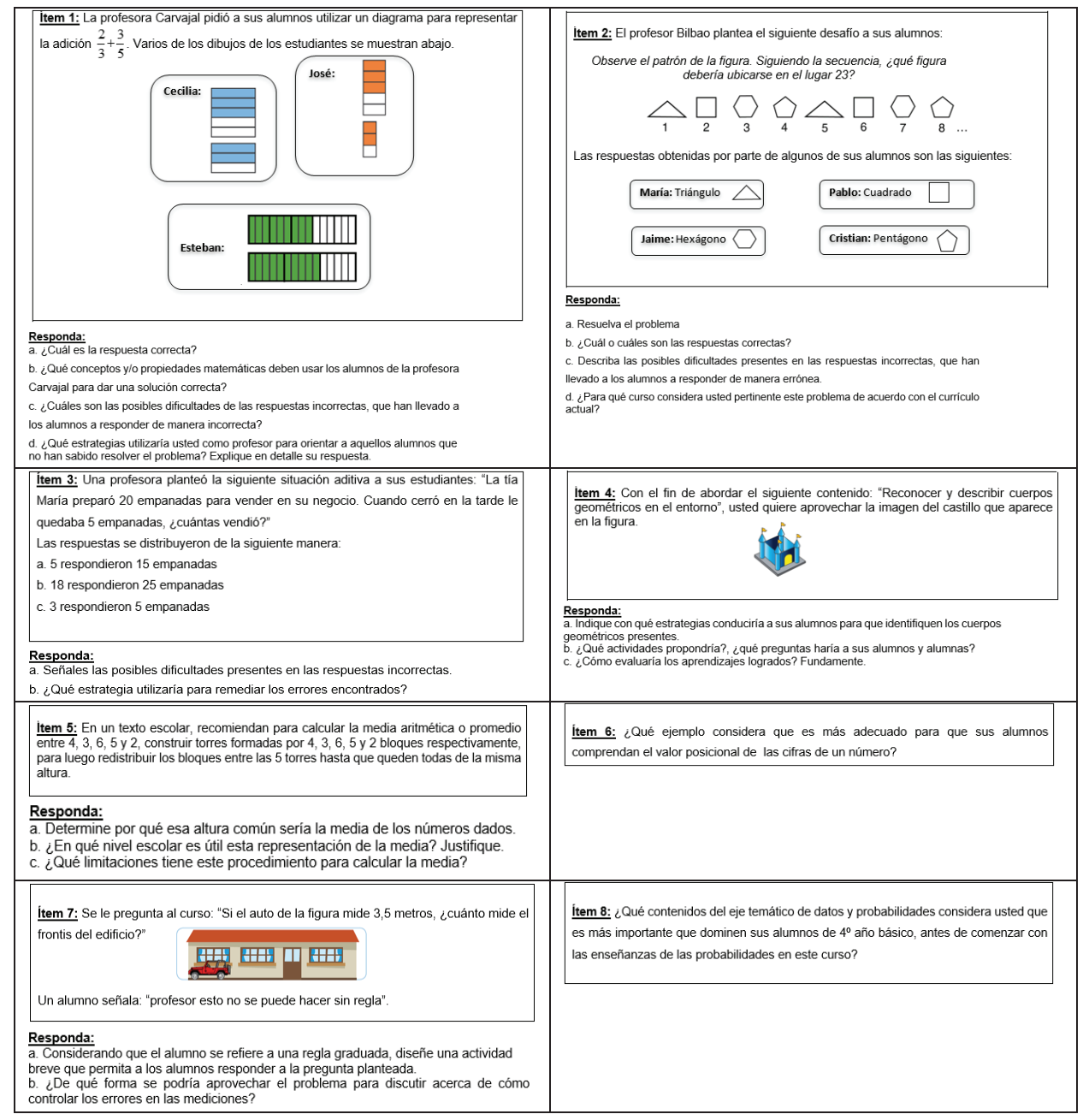


Estudios Pedagógicos XLIV, $\mathrm{N}^{\circ}$ 1: 25-48, 2018

CONOCIMIENTO DIDÁCTICO-MATEMÁTICO PARA LA ENSEÑANZA DE LA MATEMÁTICA ELEMENTAL EN

FUTUROS PROFESORES DE EDUCACIÓN BÁSICA: DISEÑO, CONSTRUCCIÓN Y VALIDACIÓN DE UN ...

\section{ANEXO 2: ÍTEMS VERSIÓN FINAL CUESTIONARIO CDM-MATEMÁTICAS ELEMENTALES}

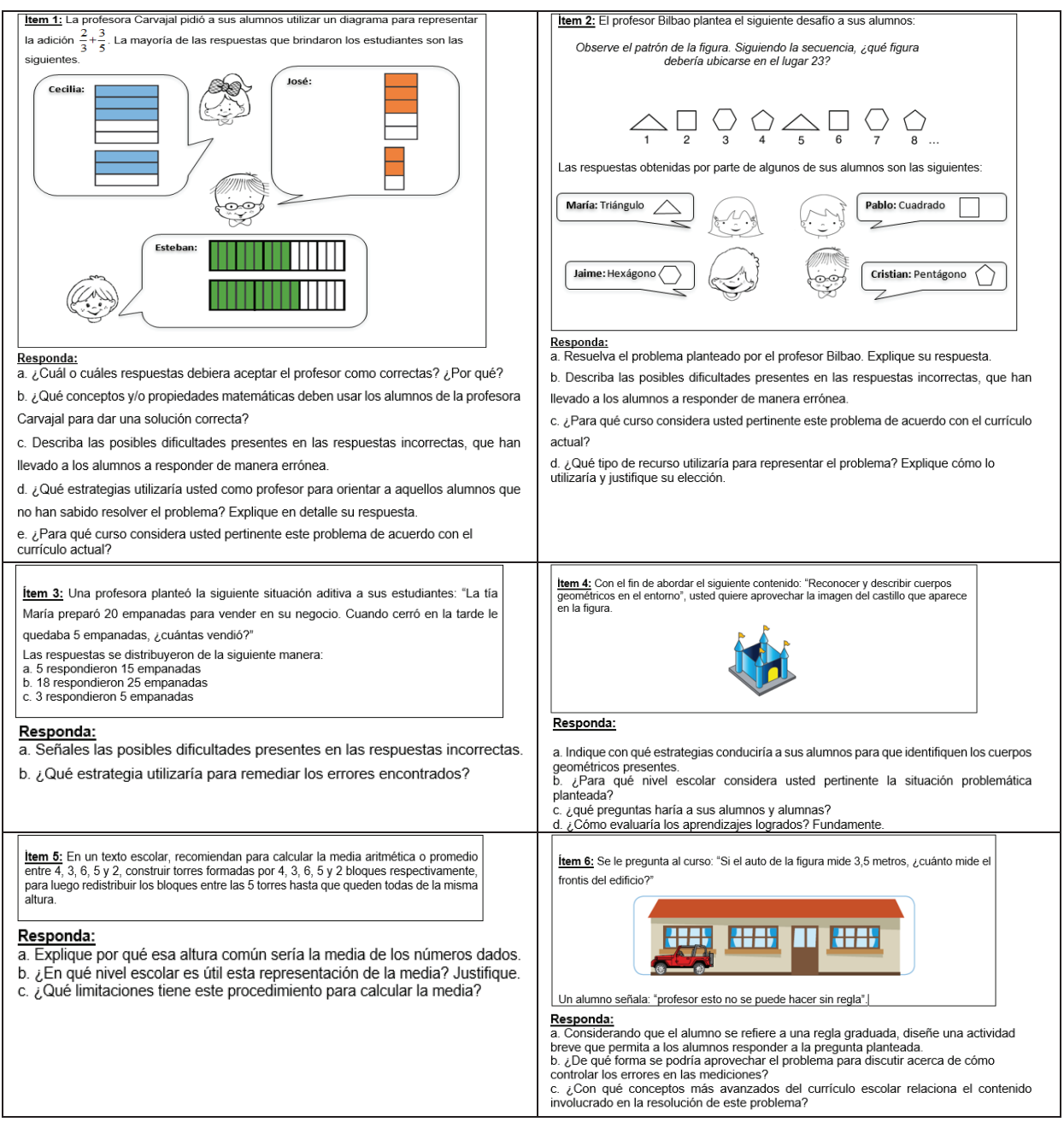

